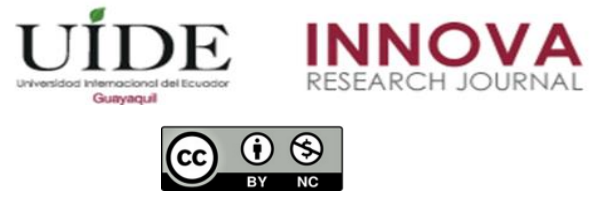

INNOVA Research Journal, ISSN 2477-9024

(Septiembre-Diciembre 2020). Vol. 5, No.3.2 pp. 214-234

DOI: https://doi.org/10.33890/innova.v5.n3.2.2020.1620

URL: http://revistas.uide.edu.ec/index.php/innova/index

Correo: innova@uide.edu.ec

\title{
Anemia, estado de salud y reinserción familiar en centros de acogida residencial del Perú, un análisis cualitativo
}

\section{Anemia, health status and family reintegration in residential care centers in Peru, a qualitative analysis}

Olga María del Carmen Ramos Huamán

(i) https://orcid.org/0000-0002-7872-3597

Universidad Norbert Wiener, Perú

Dennys Taipe Porras

(D) https://orcid.org/0000-0003-1124-0184

Katherine Aurora Chamorro Begazo

(D) https://orcid.org/0000-0003-2128-2847

Pompeyo Gabriel Asca Agama

(D) https://orcid.org/0000-0001-8819-3318

Universidad César Vallejo, Perú

Autor para correspondencia: olga.ramos@ uwiener.edu.pe; dennys.taipe.ts@ gmail.com;

katychabqw@gmail.com; pascaa@ucvvirtual.edu.pe

Fecha de recepción: 12 de octubre de 2020 - Fecha de aceptación: 04 de noviembre de 2020

\section{Resumen}

Esta investigación tuvo como objetivo general analizar el manejo de la anemia, estado de salud y la reinserción familiar en las niñas, niños y adolescentes acogidos en los Centros de Acogida Residencial del INABIF; se trabajó con el enfoque cualitativo, estudio fenomenológico que pretende precisar a través de la indagación y el análisis casos situaciones o fenómenos de un entorno específico. Se recogió la información a través de entrevistas semiestructuradas a servidores del centro de acogida residencial "Divino Jesús" que pertenece al INABIF; la entrevista estuvo compuesta por 15 preguntas guías cuyos resultados fueron posteriormente triangulados de acuerdo con el diseño del estudio. Se definieron tres categorías: manejo de la anemia; estado de salud; y reinserción familiar y social, con sus subcategorías correspondientes. Los resultados mostraron que el manejo de la anemia, estado de salud y reinserción familiar-social en las niñas, niños y adolescentes acogidos en la unidad de análisis, se llevó a cabo según lo estipulado por el ente rector, los que al ser lineamientos de salud pública genéricos y aplicables en grandes poblaciones vulnerables o no, pero sanas, no resultan aplicables en su totalidad en la población acogida en el lugar de estudio, por tener particularidades biológicas, genéticas y hasta fisiológicas que hacen necesario el manejo de la misma sea individualizada.

Palabras claves: manejo de anemia; estado de salud; reinserción familiar y social; gestión pública. 


\begin{abstract}
The general objective of this research was to analyze the management of anemia, health status and family reintegration in girls, boys and adolescents sheltered in INABIF Residential Shelter Centers; We worked with the qualitative approach, a phenomenological study that aims to clarify, through inquiry and analysis, situations or phenomena in a specific environment. The information was collected through semi-structured interviews with servers from the "Divino Jesus" residential reception center that belongs to INABIF; the interview consisted of 15 guiding questions, the results of which were later triangulated according to the study design. Three categories were defined: anemia management; health condition; and family and social reintegration, with their corresponding subcategories. The results showed that the management of anemia, health status and family-social reintegration in the girls, boys and adolescents welcomed in the unit of analysis, was carried out as stipulated by the governing body, which, being guidelines of Generic public health and applicable in large populations that are vulnerable or not, but healthy, are not applicable in their entirety in the host population at the study site, because they have biological, genetic and even physiological characteristics that make it necessary to manage it individually.
\end{abstract}

Keywords: Anemia management; health status; family and social reintegration; public management.

\title{
Introducción
}

La Organización Panamericana de la Salud, describió en estudios realizados en el año 2011 que se encontró un alto porcentaje de anemia en la población infantil, mujeres en capacidad de gestar y embarazadas en algunos países del mundo lo que significa una afectación mundial. En el 2019 reportó que en los últimos 25 años se incrementó la esperanza de vida al nacer en el continente americano; pasando de 72.3 a 76.9 años, siendo Bolivia el país con mayor aumento, seguido de las Islas Turcas y Caicos, Guatemala, Haití y Perú. La mortalidad de la niñez en la región logró disminuir de manera importante y fue considerada pionera en la eliminación de las enfermedades prevenibles mediante vacunación. En América del Sur casi 100,000 niños mueren anualmente por causas atribuidas a la violencia intrafamiliar, constituyéndose en un problema grave de la región. Situación que se hace crítica por la ausencia de estadística relacionada a niños en situación de vulnerabilidad tal como lo indicó Gonzáles (2012). Se estima que en esta región habría alrededor de 13 millones de niños sin cuidados parentales en 2003, cifra que se redujo desde 1990 sólo un 10\%. De éstos casi la totalidad son acogidos por otros familiares o dados en adopción según López (2014). A partir de esta situación las autoridades internacionales en la materia tomaron decisiones orientadas al cuidado de la población menor de edad sin protección por parte de sus padres según estableció las Naciones Unidas, (2010); el gobierno peruano emitió la ley dirigida a restituir y garantizar derechos, enfatizando el derecho a la vida, crecer y ser parte de un hogar donde goce de protección integral a población menor de 18 años en presunta desprotección.

El referente en estadística e informática nacional en el año 2018, dio a conocer que aproximadamente el $4 \%$ de la población de 3 años tenían anemia en algún grado, de estos, la mitad residen en las zonas rurales y poco menos del 50\% en la zona urbana. A partir de esto el gobierno peruano ha venido dando directrices en materia de nutrición y alimentación a través del Acuerdo Nacional - 2002, en el Plan Bicentenario (CEPLAN, 2011) y en el Plan Nacional de 
Acción por la Infancia y la Adolescencia del Sector Mujer. El sector mujer en el año 2018 acogió 1676 niños y adolescentes de ambos sexos: declarados en abandono 14\%, 85\% por Desprotección Familiar y $1 \%$ presuntos infractores. Del total de estos niños, el $28 \%$ se encontraban sanos y $72 \%$ tenía algún problema de salud, de este grupo: el $8.5 \%$ tiene desnutrición crónica, 3,9\% tiene anemia en algún grado y $0.4 \%$ desnutrición aguda.

Se considera que, en Perú, la hemoglobina baja (anemia) es un problema que afecta a una gran parte de la población, entre ellos los niños, niñas y adolescentes de las casas de acogida de la INABIF que presentan distintos grados de la enfermedad con particulares que dependen de aspectos biológicos, físicos, mentales y sociales y que son atendidos con protocolos distintos de acuerdo con los juicios que los profesionales determinan; lo que significa que no puede abordarse de manera genérica el tratamiento para cada paciente tal como dictan los protocolos y directivas del Ministerio de Salud en los programas nacionales. No tener los niveles deseados de hemoglobina afecta la salud de los niños, niñas y adolescentes y esto afectaría también su reinserción familiar - social.

Sulayyim (2019) en un estudio previo que tuvo como objetivo documentar y analizar criterios de diagnóstico y modalidades terapéuticas empleadas por los pediatras en los centros de salud afiliados al Ministerio de la Guardia Nacional, Riad, encontró algunas diferencias en el diagnóstico IDA y las prácticas terapéuticas, encontrando que en algunos casos se sugerían pruebas de laboratorios, en otras, tratamientos de hierro, en distintas dosis, etc. Cadet (2018) encontró en su estudio que la anemia por deficiencia de hierro (IDA) es el resultado de niveles bajos de hierro en el cuerpo y que la mitad de los casos con hemoglobina baja están relacionados a un déficit de hierro, concluyó que la IDA afecta a pacientes a lo largo de la vida por lo que los profesionales de salud deben determinar en cada paciente las causas, agentes o elementos considerados de riesgo para IDA y también la presentación clínica y resultados de laboratorio.

Fahima (2016) evaluó la disminución de hemoglobina por debajo de valores por carencia de hierro (IDA) en la infancia con relación a diferentes patrones de alimentación: leche materna exclusiva, leche de vaca exclusiva y leche fortificada con hierro, encontró que el $57.1 \%$ de bebés del grupo total estudiado tenían IDA; concluyó que los lactantes de 6 a 24 meses representan uno de los grupos de mayor riesgo para desarrollar la IDA (57,1\%). Díaz (2015) midió nutricionalmente a los menores de zonas originarias y no originarias de dos provincias de la zona amazónica del Perú, encontró que mediante métodos estandarizados se identificaron los factores que estarían originando alteraciones en el peso, talla y hemoglobina baja en la población de estudio. La proporción de población indígena con talla baja fue superior respecto de la no indígena, igual que la anemia. Las causas que estarían originando tales alteraciones fueron disímiles en ambas poblaciones. Predominó la edad inferior a 36 meses y características de la vivienda inadecuadas, versus pobreza extrema y el parto institucional, en indígenas y no indígenas respectivamente. Concluyeron que existen marcadas brechas entre ambas poblaciones respecto del modo de vivir de la población, disponibilidad de servicios de salud y presencia de DC y hemoglobina baja en la población infantil por lo que se requiere adecuar las intervenciones que brinda y facilita el Estado en dichas zonas.

A partir de la revisión teórica realizada, se plantearon para el presente estudio las siguientes categorías; la primera el Manejo de la anemia que es descrito por los organismos de

Esta obra se comparte bajo la licencia Creative Common Atribución-No Comercial 4.0 International (CC BY-NC 4.0) Revista de la Universidad Internacional del Ecuador. URL: https://www.uide.edu.ec/ 
salud como un procedimiento variable dependiente de las causas de origen y su nivel de gravedad. La OMS en el 2011 estableció valores de referencia para el diagnóstico de la anemia y establecer sus niveles de gravedad, para la posterior intervención de los Gobiernos para establecer políticas y estrategias para combatirla. En Costa Rica por ejemplo, se establecieron abordajes para la enfermedad en su población infantil, (Viquez, 2014); Pavo (2016) recogió en "Anemia en la edad pediátrica" la forma de manejar la anemia en niños españoles; el MINSA (2017) para el Perú diseñó la Norma Técnica - Manejo Terapéutico y Preventivo de la anemia en niños, adolescentes, mujeres gestantes y puérperas (NTS MINSA). La citada Norma Técnica define la categoría 1 Manejo de la anemia de la presente investigación en base a los productos farmacéuticos que se utilizarán contemplados en el Petitorio Único de Medicamentos (PNUME) vigente de acuerdo a esquema establecido, teniendo en cuenta el contenido del hierro elemental según cada producto farmacéutico.

Se definieron entonces las sub categorías a partir de manejo de la anemia. La primera subcategoría es el manejo preventivo de la anemia que son los procedimientos que se aplican con el fin de evitar que un paciente, de cualquier edad y condición desarrolle la enfermedad; la segunda subcategoría es manejo terapéutico de la anemia que son los procedimientos que se establecen para controlar la enfermedad presente en los pacientes, se tomó para estas subcategorías las definiciones establecidas de acuerdo con la NTS del MINSA en el 2017, este documento es el único técnico aprobado por el rector en salud del país que recoge las directrices internacionales en materia de salud, ajustados a la realidad geográfica e indicadores de salud del Perú.

La segunda categoría Estado de Salud ha evolucionado en el tiempo, definida por la OMS en la primera mitad del siglo XX como un estado completo de bienestar físico, mental y social y no solo como la ausencia de enfermedades o afecciones de cualquier tipo; lo que permitió desde esa época comprender que la saludo no solo es biológica, sino que implica valores, relaciones intra e inter personales. Kleinman (1980) interpretó los conceptos de salud y enfermedad a partir de la patología, el padecimiento y la enfermedad, términos que interrelacionan lo fisiológico, las dimensiones sociales de la persona, respuestas afectivas y procesos cognitivos, los que hacen que a partir de ese análisis la salud pueda ser definida como un hecho social, alrededor de esta, se desarrollan respuestas médicas locales y se construyen diferentes sistemas salud que apuntan a atender sus necesidades. En este estudio se escogió la anemia como la enfermedad analizada en este estudio. De acuerdo el Ministerio de Salud (2018), el estado de salud coincide con la definición de Kleinman.

Las Sub categorías de la categoría 2 según el MINSA (2018) fueron definidas según los determinantes de la salud, nombre colectivo con el que se nombra a los factores y condiciones que influyen en la salud de las poblaciones y considera los recogidos en el modelo de Marc Lalonde donde están identificados cuatro determinantes básicos. Según el Instituto Nacional de Salud (2018), estos se definen como: (a) entorno que circunscribe aspectos extraños al propio cuerpo, sobre los cuales la persona tiene una restringida capacidad de control y que influyen sobre la salud. Medio ambiente físico, medio ambiente social; (b) estilos de vida, los comportamientos de los individuos y las colectividades ejercen una influencia sobre su salud. Los hábitos de vida nocivos (consumo de drogas, nutrición inadecuada-sedentarismo y otros) tienen efectos nocivos para la salud; (c) biología humana, circunscribe aspectos físicos y 
mentales que integran la corporalidad humana, como son la herencia genética, la maduración y envejecimiento, particularidades de los sistemas del cuerpo (nervioso, endocrino, digestivo); (d) servicios sanitarios, la cantidad y calidad de recursos utilizados con la finalidad de satisfacer las carencias y reclamos de los ciudadanos. Asimismo, entendemos a las estructuras, organizaciones y disposiciones generales del sector salud.

Arredondo (1992), el análisis de modelos teóricos que abordan como mismo objeto de estudio y desde diferentes disciplinas el proceso salud-enfermedad, incluyó once propuestas, las que se enuncian a continuación: i) Modelo Mágico-Religioso, ii) Modelo Sanitarista, iii) Modelo Social, iv) Modelo Unicausal, v) Modelo Multicausal, vi) Modelo Epidemiológico, vii) Modelo Ecológico, viii) Modelo Histórico-Social, ix) Modelo Geográfico, x) Modelo Económico y xi) Modelo Interdisciplinario.

La tercera categoría del estudio fue la reinserción familiar y social sustentada en lo asumido en la Convención de las Naciones Unidas que reunida en 1989 que creó un tratado sobre los derechos humanos del niño, resultando en una versión ampliada de la Declaración Universal de los Derechos Humanos del Niño escrita en 1959. En este tratado se define la obligación de los estados deberán velar porque los niños no sean separados de sus padres contra su voluntad a menos que la ley lo determine; así mismo deben prestar la asistencia apropiada a los padres y a los representantes legales para el desempeño de sus funciones en lo que respecta a la crianza del niño y velar por la creación de instituciones, instalaciones y servicios para el cuidado de los niños.

El Decreto Legislativo $N^{\circ} 1297$ del sector rector para la Protección de los menores de edad sin cuidados parentales o en riesgo de perderlos y su Reglamento aprobado con Decreto Supremo No 001-2018-MIMP, señaló las disposiciones de protección que involucren o determinen la separación familiar, y que el Estado orienta su accionar a la integración familiar, con la implementación de acciones y programas enfocados al apoyo y facilitar el regreso de los menores de edad al seno familiar de origen. Tal circunstancia de retorno al seno familiar de origen permite la valoración positiva precedente y el consecuente acomodo del menor de edad con la intervención de los integrantes de la familia nuclear y equipo técnico que asumió su acogimiento provisional. En ese sentido, involucra el acompañar con la finalidad de extender la orientación y apoyo que requiera, en cumplimiento del plan de trabajo individual.

Las sub categorías de la variable Reinserción familiar se sustentaron en lo establecido en el artículo 89 del D.L. $\mathrm{N}^{\circ}$ 1297, las que se enuncian a continuación: (a) desaparición de las circunstancias que dieron origen a la protección; (b) disposición de la autoridad judicial; y (c) cumplimiento de la mayoría de edad de la persona acogida.

Se definió como marco espacial de la presente investigación al INABIF y se analizó el manejo de la anemia, en los CAR a su cargo, estado de salud y reinserción social, siendo el marco temporal de desarrollo de la investigación, el año 2020 en convivencia con la pandemia producida por la presencia de la COVID-19, el Estado de Emergencia Sanitaria en el país y el aislamiento social obligatorio determinado por el gobierno en el período marzo-junio 2020. La relevancia de este estudio se justifica en el análisis del modelo actual de atención para el manejo de la anemia en los menores de edad, siendo esta una enfermedad presente en una gran parte de 
la población peruana; se espera que los resultados obtenidos permitan a los funcionarios que correspondan, hagan los cambios que permitan mejorar los protocolos de atención de la enfermedad lo que afectaría positivamente el estado de salud y la reinserción familiar de este sector de la población (Balcázar y otros, 2013).

Se plantearon las siguientes preguntas de investigación; como principal: ¿Cómo es el manejo de la anemia, estado de salud y reinserción familiar - social en las niñas, niños y adolescentes acogidos en los Centros de Acogida Residencial del INABIF?, a partir de esta primera pregunta, se definieron preguntas específicas: ¿Cómo es el manejo de la anemia en el entorno de las niñas, niños y adolescentes acogidos en los Centros de Acogida Residencial del INABIF?. ¿Cómo es el manejo de la anemia en el estilo de vida de las niñas, niños y adolescentes acogidos en los Centros de Acogida Residencial del INABIF?. ¿Cómo es el manejo de la anemia en la biología humana de las niñas, niños y adolescentes acogidos en los Centros de Acogida Residencial del INABIF?. ¿Cómo es el manejo de la anemia en los servicios sanitarios que reciben las niñas, niños y adolescentes acogidos en los Centros de Acogida Residencial del INABIF?. ¿Cómo es el manejo de la anemia en el proceso de reinserción familiar - social de las niñas, niños y adolescentes acogidos en los Centros de Acogida Residencial del INABIF?.

Estas preguntas generaron los siguientes objetivos: objetivo principal: analizar el manejo de la anemia, estado de salud y reinserción familiar en las niñas, niños y adolescentes acogidos en los Centros de Acogida Residencial del INABIF; objetivos específicos: Analizar el manejo de la anemia en el entorno de las niñas, niños y adolescentes acogidos en los Centros de Acogida Residencial del INABIF. Analizar el manejo de la anemia en el estilo de vida de las niñas, niños y adolescentes acogidos en los Centros de Acogida Residencial del INABIF. Analizar el manejo de la anemia en la biología humana de las niñas, niños y adolescentes acogidos en los Centros de Acogida Residencial del INABIF. Analizar el manejo de la anemia en la biología humana de las niñas, niños y adolescentes acogidos en los Centros de Acogida Residencial del INABIF. Analizar el manejo de la anemia en el proceso de reinserción familiar de las niñas, niños y adolescentes acogidos en los Centros de Acogida Residencial del INABIF.

\section{Metodología}

El tipo de estudio aplicado es básico; de paradigma interpretativo, (Martínez, 2006; Quecedo y Castaño, 2002; Ricoy, 2006) El enfoque, fue cualitativo, su diseño fue a través del estudio de caso, estudio empírico fenomenológico social, que permitió comprender a partir de la observación crítica y reflexiva hechos reales en el medio dónde se desarrollan las actividades; método inductivo (Chaverra, Gaviria, y González, 2019; Hernández y otros, 2014; Martínez, 1987; Naupas y otros, 2014; Ugalde y Balbastre, 2013).

Los participantes se seleccionaron de forma deliberada e intencional (Rodríguez, Gil y García, 1999, p. 135). Se hizo de forma individual con los niveles que concordaban con las características o particularidades definidas en el diseño (Hernández y otros, 2014) Los sujetos de estudio fueron los siguientes: (a) directoras de los centros de acogida residencial: encargados de gerenciar los CAR; (b) personal de salud: profesional galeno, enfermera y técnico, que tienen como labor fundamental estabilizar la salud de los residentes; así como, atenderlos en caso de 
urgencia y emergencia; (c) nutricionista: profesional de salud que tiene a cargo labores administrativas, de índole clínico y además tiene a cargo la programación de la alimentación de los residentes del CAR; (d) personal administrativo: encargado de brindar atención oportuna ante requerimientos esenciales de los usuarios o residentes; y (e) equipo técnico: conformado por un staff de profesionales de las unidades orgánicas que desarrollan tareas y roles específicos, que avalan el trabajo interdisciplinario coordinado y efectivo.

\section{Tabla 1}

Codificación de los sujetos participantes en la investigación

\begin{tabular}{lcc}
\multicolumn{1}{c}{ Sujetos } & Letras & Código \\
\hline Directoras de CAR & D & D1 \\
Personal de Salud & S & S1 \\
Nutricionista & N & N1 \\
Personal administrativo & A & A1 \\
Personal del equipo técnico & T & T1 \\
Nota: Participantes involucrados en la investigación (2020). Elaboración propia.
\end{tabular}

Las técnicas utilizadas para el levantamiento de información fueron: a) Revisión documental, importante en la etapa exploratoria que permite describir el objeto de investigación; b) Entrevista, diálogo donde fluye el intercambio de preguntas y respuestas. (Ander-Egg, 2014; Baena, 1985; Carrasco, 2019; Denzin y Lincoln, 2005; Hernández y otros, 2014; Naupas y otros, 2014; Ruiz, 1999; Sánchez y Reyes, 2006; Solis, 2003).

Los instrumentos utilizados fueron la matriz de análisis documental que permite detectar, obtener y consultar la bibliografía y otros materiales para los propósitos del estudio; se utilizaron documentos normativos, decretos legislativos, información bibliográfico y libros relacionados, artículos de revistas indexadas relacionadas con el tema de estudio (Hernández y otros, 2000). Adicional se utilizó una entrevista estructurada a profundidad que permitió el diálogo entre las partes participantes escogidas previamente: i) Directora del CAR, ii) Personal de Apoyo Permanente, iii) Profesional de salud, iv) Nutricionista, v) Personal administrativo, vi) Personal del equipo técnico y iii) familiares de los NNA acogidos en el CAR. La entrevista se fundamentó en pautas de cuestionamientos del entrevistador, quien tuvo la autonomía de añadir cuestiones para clarificar conceptos u conseguir mayor información, la guía de preguntas fue validada por expertos en el campo de la niñez y adolescencia (Acevedo y López, 1986; Hernández, Fernández y Baptista, 2014).

En ese marco, en la presente investigación se recabó información sobre la normativa vigente de los CAR del INABIF, Manuales de atención, Guías, Protocolos; así como toda la bibliografía relacionada al manejo de anemia en los usuarios. El diseño e instrumento de 
investigación fue sujeto a revisión de expertos y fue diligenciado en los sujetos de estudio ya indicados. Con la información recopilada se procedió a la desgrabación de las entrevistas en las matrices respectivas codificando las respuestas para la sistematización. Se tabuló la información obtenida por medio de los instrumentos. Se analizaron las narraciones y obtuvieron resultados. Con los resultados y a efectos de analizar la información obtenida con diferentes ópticas se convocó a un equipo multidisciplinario de expertos en el tema, a fin de efectuar un mejor y enriquecido análisis. (Ñaupas, Mejía, Novoa y Villagómez, 2014).

Finalmente, como fruto de las acciones realizadas en campo se presentaron las categorías apriorísticas y emergentes, en las tablas dos, tres, cuatro y cinco se presentan las matrices de categorización y subcategorización de las categorías:

\section{Tabla 2}

\section{Matriz de categorización}

\begin{tabular}{|c|c|c|c|}
\hline \multirow{4}{*}{\begin{tabular}{l} 
Problema general \\
\multicolumn{2}{c}{ ¿Cómo es el manejo } \\
de la anemia, estado \\
de salud y reinserción \\
familiar-social en las \\
niñas, niños \\
adolescentes \\
acogidos en los \\
Centros de Acogida \\
Residencial \\
INABIF?
\end{tabular}} & \multirow{4}{*}{$\begin{array}{l}\text { Objetivo general } \\
\text { Analizar el manejo de la } \\
\text { anemia, estado de salud y } \\
\text { reinserción famlliar-social en } \\
\text { las niñas, niños y adolescentes } \\
\text { acogidos en los Centros de } \\
\text { Acogida Residencial del } \\
\text { INABIF. }\end{array}$} & \multicolumn{2}{|c|}{ Categorías / Indicadores } \\
\hline & & \multicolumn{2}{|c|}{ Categoría 1: Manejo de la anemia } \\
\hline & & Sub Categorías & Indicadores \\
\hline & & \multirow[t]{3}{*}{ Manejo Preventivo } & $\begin{array}{l}\text { Suplementación preventiva } \\
\text { con hierro y micronutrientes } \\
\text { para niños menores de } 36 \text { meses }\end{array}$ \\
\hline $\begin{array}{r}\text { Problema } \\
\text { específico } 1 \\
\end{array}$ & Objetivo específico 1 & & $\begin{array}{c}\text { Suplementación preventiva } \\
\text { para las adolescentes mujeres de }\end{array}$ \\
\hline $\begin{array}{l}\text { ¿Cómo es el manejo } \\
\text { de la anemia en el }\end{array}$ & $\begin{array}{c}\text { Analizar el manejo de la } \\
\text { anemia en el entorno de las }\end{array}$ & & 12 a 17 años \\
\hline $\begin{array}{l}\text { entorno de las niñas, } \\
\text { niños y adolescentes } \\
\text { acogidos en los } \\
\text { Centros de Acogida } \\
\text { Residencial r del } \\
\text { INABIF? }\end{array}$ & $\begin{array}{l}\text { niñas, niños y adolescentes } \\
\text { acogidos en los Centros de } \\
\text { Acogida Residencial del } \\
\text { INABIF }\end{array}$ & \multirow[t]{4}{*}{$\begin{array}{c}\text { Manejo } \\
\text { Terapéutico }\end{array}$} & $\begin{array}{l}\text { Tratamiento con hierro para } \\
\text { niños prematuros y/o con bajo } \\
\text { peso al nacer menores de } 6 \\
\text { meses de edad con anemia }\end{array}$ \\
\hline $\begin{array}{r}\text { Problema } \\
\text { específico 2 }\end{array}$ & Objetivo específico 2 & & $\begin{array}{l}\text { Tratamiento con hierro para } \\
\text { niños peso al nacer menores de }\end{array}$ \\
\hline \multirow{2}{*}{$\begin{array}{l}\text { Cspecífico 2 } \\
\text { ¿Cómo es el manejo } \\
\text { de la anemia en el } \\
\text { estilo de vida de las } \\
\text { niñas, niños y } \\
\text { adolescentes } \\
\text { acogidos en los } \\
\text { Centros de Acogida } \\
\text { Residencial } \\
\text { INABIF? }\end{array}$} & \multirow{2}{*}{$\begin{array}{l}\text { Analizar el manejo de la } \\
\text { anemia en el estilo de vida de } \\
\text { las niñas, niños y adolescentes } \\
\text { acogidos en los Centros de } \\
\text { Acogida Residencial del } \\
\text { INABIF }\end{array}$} & & $\begin{array}{l}6 \text { meses a } 11 \text { años de edad con } \\
\text { anemia leve o moderada }\end{array}$ \\
\hline & & & $\begin{array}{l}\text { Tratamiento de la anemia en } \\
\text { varones y mujeres adolescentes } \\
\text { de } 12 \text { a } 17 \text { años de edad con } \\
\text { anemia leve y moderada }\end{array}$ \\
\hline $\begin{array}{r}\text { Problema } \\
\text { específico } 3\end{array}$ & Objetivo específico 3 & \multicolumn{2}{|c|}{ Categoría 2: Estado de salud } \\
\hline \multirow{2}{*}{$\begin{array}{l}\text { ¿Cómo es el } \\
\text { manejo de la anemia }\end{array}$} & \multirow{2}{*}{$\begin{array}{l}\text { Analizar el manejo de la } \\
\text { anemia en la biología humana }\end{array}$} & Entorno & Medio ambiente físico \\
\hline & & & Medio ambiente social \\
\hline
\end{tabular}




\begin{tabular}{|c|c|c|c|}
\hline Problema general & Objetivo general & \multicolumn{2}{|c|}{ Categorías / Indicadores } \\
\hline \multirow{2}{*}{$\begin{array}{l}\text { en la biología } \\
\text { humana de las niñas, } \\
\text { niños y adolescentes } \\
\text { acogidos en los } \\
\text { Centros de Acogida } \\
\text { Residencial del } \\
\text { INABIF? }\end{array}$} & \multirow{2}{*}{$\begin{array}{l}\text { de las niñas, niños y } \\
\text { adolescentes acogidos en los } \\
\text { Centros de Acogida } \\
\text { Residencial del INABIF }\end{array}$} & Estilos de vida & $\begin{array}{l}\text { Nutrición inadecuada- } \\
\text { sedentarismo }\end{array}$ \\
\hline & & \multirow[t]{2}{*}{ Biología humana } & Herencia genética \\
\hline $\begin{array}{r}\text { Problema } \\
\text { específico } 4 \\
\end{array}$ & Objetivo específico 4 & & Sistema inmunológico \\
\hline \multirow{4}{*}{$\begin{array}{c}\text { ¿Cómo es el } \\
\text { manejo de la anemia } \\
\text { en los servicios } \\
\text { sanitarios que } \\
\text { reciben las niñas, } \\
\text { niños y adolescentes } \\
\text { acogidos en los } \\
\text { Centros de Acogida } \\
\text { Residencial del } \\
\text { INABIF? }\end{array}$} & \multirow{4}{*}{$\begin{array}{l}\text { Analizar el manejo de la } \\
\text { anemia en los servicios } \\
\text { sanitarios que reciben las } \\
\text { niñas, niños y adolescentes } \\
\text { acogidos en los Centros de } \\
\text { Acogida Residencial del } \\
\text { INABIF }\end{array}$} & Servicios sanitarios & $\begin{array}{l}\text { Cantidad de recursos } \\
\text { utilizados }\end{array}$ \\
\hline & & & Calidad de recursos utilizados \\
\hline & & & Política de salud del CAR \\
\hline & & \multicolumn{2}{|c|}{ Categoría 3: Reinserción familiar y social } \\
\hline $\begin{array}{r}\text { Problema } \\
\text { específico } 5\end{array}$ & Objetivo específico 5 & $\begin{array}{l}\text { Desaparición de las } \\
\text { circunstancias que } \\
\text { dieron origen a la } \\
\text { protección }\end{array}$ & $\begin{array}{l}\text { Adecuado desempeño de los } \\
\text { deberes de cuidado y protección } \\
\text { por parte de los responsables } \\
\text { del cuidado de las niñas, niños } \\
\text { y adolescentes }\end{array}$ \\
\hline \multirow{3}{*}{$\begin{array}{l}\text { ¿Cómo es el } \\
\text { manejo de la anemia } \\
\text { en el proceso de } \\
\text { reinserción familiar - } \\
\text { social de las niñas, } \\
\text { niños y adolescentes } \\
\text { acogidos en los } \\
\text { Centros de Acogida } \\
\text { Residencial del } \\
\text { INABIF? }\end{array}$} & \multirow{3}{*}{$\begin{array}{l}\text { Analizar el manejo de la } \\
\text { anemia en el proceso de } \\
\text { reinserción familiar de las } \\
\text { niñas, niños y adolescentes } \\
\text { acogidos en los Centros de } \\
\text { Acogida Residencial del } \\
\text { INABIF }\end{array}$} & $\begin{array}{l}\text { Disposición de la } \\
\text { autoridad judicial }\end{array}$ & $\begin{array}{l}\text { Preparación previa a su } \\
\text { egreso }\end{array}$ \\
\hline & & $\begin{array}{l}\text { Cumplimiento de la } \\
\text { mayoría de edad de } \\
\text { la persona acogida }\end{array}$ & Plan de vida \\
\hline & & & $\begin{array}{l}\text { Redes familiares y sociales } \\
\text { fortalecidas }\end{array}$ \\
\hline
\end{tabular}

\section{Tabla 3}

Matriz categorización de manejo de la anemia

\begin{tabular}{ll}
\hline \multicolumn{1}{c}{ Sub categoría } & Indicadores \\
\hline Manejo Preventivo & $\begin{array}{l}\text { Suplementación preventiva con hierro y micronutrientes } \\
\text { para niños menores de } 36 \text { meses. }\end{array}$ \\
& $\begin{array}{l}\text { Suplementación preventiva para las adolescentes mujeres } \\
\text { de } 12 \text { a } 17 \text { años. }\end{array}$ \\
& Tratamiento con hierro para niños prematuros y/o con bajo \\
peso al nacer menores de 6 meses de edad con anemia. & Tratamiento con hierro para niños peso al nacer menores de \\
& 6 meses a 11 años de edad con anemia leve o moderada. \\
& Tratamiento de la anemia en varones y mujeres adolescentes \\
de 12 a 17 años de edad con anemia leve y moderada.
\end{tabular}




\section{Tabla 4}

Matriz categorización del estado de salud

\begin{tabular}{ll}
\hline Sub categoría & Indicadores \\
\hline Entorno & Medio ambiente físico \\
& Medio ambiente social \\
Estilos de vida & Nutrición inadecuada-sedentarismo \\
Biología humana & Herencia genética \\
& Sistema inmunológico \\
Servicios sanitarios & Cantidad de recursos utilizados \\
& Calidad de recursos utilizados \\
& Política de salud del CAR \\
\hline
\end{tabular}

\section{Tabla 5}

Matriz categorización de reinserción familiar y social

\begin{tabular}{ll}
\hline Sub categoría & Indicadores \\
\hline $\begin{array}{l}\text { Desaparición de las circunstancias que dieron } \\
\text { origen a la protección }\end{array}$ & $\begin{array}{l}\text { Adecuado desempeño de los deberes de } \\
\text { cuidado y protección por parte de los } \\
\text { responsables del cuidado de las niñas, niños y } \\
\text { adolescentes }\end{array}$ \\
$\begin{array}{l}\text { Disposición de la autoridad judicial } \\
\begin{array}{l}\text { Cumplimiento de la mayoría de edad de la } \\
\text { persona acogida }\end{array}\end{array}$ & $\begin{array}{l}\text { Preparación previa a su egreso vida } \\
\end{array}$ \\
& Redes familiares y sociales fortalecidas
\end{tabular}

Una vez levantada la información se procedió a su codificación y análisis; todos los participantes participaron de forma honesta y voluntaria.

\section{Resultados}

El análisis de los resultados de la investigación se presenta en relación a la triangulación de las respuestas de las entrevistas aplicadas a los participantes seleccionados, técnica donde se usan tres o más perspectivas, o varias fuentes de datos; a partir de la información obtenida de los 
participantes se construyó la matriz de triangulación, cuyos resultados generales interpretados se exponen a continuación:

De acuerdo a: objetivo específico 1: Analizar el manejo de la anemia en el entorno de las niñas, niños y adolescentes acogidos en los Centros de Acogida Residencial del INABIF. Para responder a este objetivo se realizaron las siguientes preguntas:

¿Tiene usted conocimiento de cómo determinan el tipo de suplementación que se le entregará a la niña, niño y adolescente en el Centro de Acogida Residencial, a partir de los resultados del tamizaje de anemia que se les aplica? Explique y detalle si toman de referencia algún protocolo o normativa. Los entrevistados manifestaron que el manejo de la anemia en los CAR del INABIF se realizó tomando en cuenta la normativa del Ministerio de Salud, aunque no todos los niveles jerárquicos y distintos profesionales la conozcan a detalle. Según lo respondido por los entrevistados el Establecimiento de salud hace el tamizaje y determina como va a llevarse a cabo el manejo de la anemia de resultar algún usuario con algún tipo de anemia. En el rector lo dispuesto en la antes señalada normativa no es aplicado en los mismos términos por todos los Departamentos y profesionales, los pediatras tienen un criterio (cortan el tratamiento al haberse superado la anemia) y en Crecimiento y Desarrollo (CRED) las enfermeras tienen otro (brindan el tratamiento por 06 meses). En el CAR si hay disponibilidad del suplemento se administra a los usuarios, si no se cuenta con stock Inabif no los adquiere. El seguimiento a la cadena de suministro lo realiza la enfermera no el médico, éste último no atiende anemia, sólo atiende patologías. La nutricionista vigila la administración de la suplementación, al igual que las tutoras.

¿Tiene usted conocimiento de cómo entregan la suplementación de tipo preventivo a las niñas y niños menores de 36 meses acogidos en el Centro de Acogida Residencial? Los entrevistados manifestaron que la suplementación de tipo preventivo a los infantes acogidos en el CAR se realiza a través de la entrega de suplementos tipo micronutrientes, los que son entregados por el Establecimiento de Salud, a partir de ello la enfermera entrega los suplementos al servicio de Nutrición, quienes a su vez suministran a las tutoras para el consumo de los bebes. No todos los niveles jerárquicos y distintos profesionales conocen a detalle la forma de suplementar a este grupo etáreo.

¿Sabe usted cómo entregan la suplementación terapéutica a los niños prematuros y/o con bajo peso al nacer menores de edad con anemia, de 6 meses a 11 años de edad con anemia leve o moderada y varones y mujeres adolescentes de 12 a 17 años de edad con anemia leve y moderada en el Centro de Acogida Residencial? Los entrevistados manifestaron que la suplementación de tipo terapéutica a los niños que llegaron a los hogares con antecedente de haber nacido antes de tiempo y/o con bajo peso al nacer con anemia, los niños de 6 meses a 11 años de edad con anemia leve o moderado y adolescentes de ambos sexos hasta antes de cumplir mayoría de edad con anemia leve y moderada acogidos en los CAR se realiza a través de suplementos tipo sulfato ferroso, entregados por el MNSA. Los niveles jerárquicos y distintos profesionales no conocen a detalle la forma de suplementar a este grupo etáreo. Si se rompe la cadena de suministro el CAR no los adquiere. Según las indicaciones del profesional del Establecimiento de Salud, el menor es llevado al control respectivo, donde el pediatra ante valores normales de hemoglobina, suspende la suplementación. 
¿Usted considera que tanto el medio físico como el medio ambiente social del Centro de Acogida Residencial resultan beneficiosos para el estado de salud de las niñas, niños y adolescentes del CAR, cuando éstos tienen anemia? ¿Por qué? Los entrevistados manifestaron que el entorno físico, como el medio ambiente social de los CAR es beneficioso para el estado de salud de los usuarios, cuando éstos tienen anemia, puesto que allí tienen un equipo técnico dedicado a ellos que organizan sus actividades para recuperar su salud. Asimismo, señalan disponen de infraestructuras que han sido adecuadas para un mejor desarrollo. No obstante, requieren que las familias formen parte del proceso recuperativo y no solo el entorno social esté integrado por personal del CAR. Asimismo, los entrevistados han manifestado que ante la falta del apoyo de la familia algunas condiciones del entorno social no se dan en la medida de lo esperado, por lo que manifiestan superada la anemia el residente debe ser reinsertado con su familia, a fin de que cuente con el soporte emocional adecuado que soporte su crecimiento.

Finalmente, los entrevistados manifestaron que el manejo de la anemia en los CAR tanto preventiva como terapéutica se realiza tomando en cuenta la normativa del MINSA y es suministrada por ellos. A su vez en el rector la antes citada normativa no es aplicada en los mismos términos por todos los profesionales, encontrándose grandes diferencias entre pediatras y enfermeras. Si el suministro se rompe Inabif no los adquiere. La suplementación de tipo preventivo es con micronutrientes y la terapéutica es con sulfato ferroso. Asimismo, los entrevistados manifestaron que el entorno físico, como el medio ambiente social de los usuarios es beneficioso para el estado de salud de los mismos cuando éstos tienen anemia, puesto que allí tienen un equipo técnico dedicado a ellos que organizan sus actividades para recuperar su salud, disponen de infraestructuras que han sido adecuadas para un mejor desarrollo y en lo social las trabajadoras sociales los comunican con sus familias a fin de que fortalezcan sus lazos y apoyen en la recuperación como parte del proceso de reinserción en la familia nuclear o familia extensa.

Objetivo específico 2: Analizar el manejo de la anemia en el estilo de vida de las niñas, niños y adolescentes acogidos en los Centros de Acogida Residencial del INABIF. Para responder a este objetivo se realizaron las siguientes preguntas:

¿Usted percibe que la alimentación que se brinda en el CAR, es balanceada, suficiente, variada y goza de aceptabilidad en las niñas, niños y adolescentes del CAR? Detalle. Los entrevistados manifestaron que la alimentación que se brinda en el CAR va a estar diferenciada por la presencia o no de un profesional nutricionista. La planificación es acorde a las necesidades de los usuarios, pudiendo ser programada por el nutricionista del Establecimiento de Salud o del CAR. Asimismo, refieren que es notoria la ausencia del profesional nutricionista a tiempo completo en el CAR durante el acompañamiento y asistencia técnica al personal de cocina durante el servido de las raciones, a nivel de calidad alimentaria y de porciones servidas. No obstante ello, al aplicar encuestas que miden la aceptación de las raciones y satisfacción de los residentes, los resultados son satisfactorios en la mayoría de ellos.

¿Tiene usted conocimiento si las niñas, niños y adolescentes realizan actividad física en el CAR? ¿De ser afirmativa la respuesta, considera que es beneficioso para su salud? Los entrevistados manifestaron que los usuarios realizan actividad física en el CAR, aprovechando la estructura de los mismos, realizan las actividades en ocasiones con profesores contratados para tal fin. Asimismo, manifiestan que es beneficioso para la salud de los usuarios considerando que

Esta obra se comparte bajo la licencia Creative Common Atribución-No Comercial 4.0 International (CC BY-NC 4.0) Revista de la Universidad Internacional del Ecuador. URL: https://www.uide.edu.ec/ 
regula su nivel hormonal, liberan estrés, les hace gastar energías y ayuda a mantener un peso saludable, actividad en la que no son incluidos los menores de 3 años.

Finalmente, los entrevistados manifestaron que el manejo de la anemia en el estilo de vida de los usuarios, respecto de la alimentación es acorde a sus necesidades, planificada con la participación de un profesional nutricionista del CAR o Establecimiento de Salud. Identifican debilidad en el acompañamiento y asistencia técnica al personal de cocina durante el servido de las raciones, a nivel de calidad alimentaria y de porciones servidas. Las encuestas de satisfacción aplicadas muestran resultados satisfactorios en la mayoría de ellos. Asimismo, los entrevistados señalaron que complementariamente a la alimentación, los usuarios realizan actividad física bajo las indicaciones de profesores contratados para tal fin, aprovechando la infraestructura que disponen para regular el nivel hormonal, estrés, mantener un peso saludable y llevar un estilo de vida saludable actividad en la que no son incluidos los menores de 3 años.

Objetivo específico 3: Analizar el manejo de la anemia en la biología humana de las niñas, niños y adolescentes acogidos en los Centros de Acogida Residencial del INABIF. Para responder a este objetivo se realizaron las siguientes preguntas:

¿Usted considera que la herencia genética y sistema inmunológico de cada niña, niño y adolescente acogido en el CAR debe ser tomado en cuenta para el manejo de la anemia y/o suplementación que va a recibir? Explique. Los entrevistados manifestaron que la herencia genética y sistema inmunológico debe ser tomado en cuenta para el manejo de la anemia y/o suplementación que va a recibir los usuarios, considerando que ellos tienen antecedentes familiares diversos referidos a consumo de drogas, alcohol, enfermedades psiquiátricas, que afectan sus sistemas inmunológicos y digestivos, los que podrían estar originando que la anemia aparezca de manera recurrente. Según los entrevistados el CAR aplica el protocolo dispuesto por el Ministerio de Salud teniendo en cuenta solamente el criterio referido a presencia de anemia, los otros factores no son considerados, infiriendo que estos podrían repercutir en el estado nutricional futuro del niño, ya que este no tendrá las reservas suficientes de hierro para enfrentar la vida extrauterina y requerirá una suplementación preventiva temprana.

Finalmente, los entrevistados manifestaron que el manejo de la anemia en la biología humana de los usuarios, desde la perspectiva de la herencia genética es diversa y va a depender de los antecedentes de la madre desde la gestación, factores culturales, idiosincrasia de la familia de origen y hábitos no saludables, la que no es abordada en los CAR según sus individualidades y atendiendo sus particularidades. Asimismo, la biología humana respecto del sistema inmunológico de cada uno ellos responden de manera diferente en concordancia a la alimentación, forma de vida y situaciones a las que han sido expuestos desde la vida intrauterina, aspectos que no son tomados en cuenta en el manejo de la anemia en los usuarios.

Objetivo específico 4: Analizar el manejo de la anemia en los servicios sanitarios que reciben las niñas, niños y adolescentes acogidos en los Centros de Acogida Residencial del INABIF. Para responder a este objetivo se realizaron las siguientes preguntas:

¿Usted percibe que los recursos humanos utilizados en la intervención efectuada por el área de salud del CAR se ajustan a las necesidades de las niñas, niños y adolescentes y atienden sus respectivas particularidades? Explique. Los entrevistados manifestaron que los recursos 
humanos utilizados en la intervención efectuada por el área de salud del CAR no se ajustan a las necesidades de los usuarios, ni atienden sus respectivas particularidades, considerando que no hay cantidad suficiente de profesionales y que además estos no están capacitados para atender las diferentes problemáticas de todos los usuarios. Algunos CAR cuentan con equipo técnico completo y otros no, por lo que la normativa interna del Inabif los faculta a recurrir a la atención que brindan los Establecimientos del Primer Nivel de Atención del MINSA, recibiendo atenciones no especializadas. Adicional a ello, el rector ha establecido prioridades en sus atenciones, por lo que los CAR que tienen a cargo a niños mayores de 03 años y adolescentes varones nunca serán atendidos de manera prioritaria, aunque así lo requieran.

¿Usted percibe que los recursos financieros utilizados en la intervención efectuada por el área de salud del CAR se ajustan a las necesidades de las niñas, niños y adolescentes y atienden sus respectivas particularidades? Explique. Los entrevistados manifestaron que los recursos financieros utilizados en la intervención efectuada por el área de salud del CAR no se ajustan a las necesidades de los usuarios, ni atienden sus respectivas particularidades, ya que en muchos casos estos no cubren la totalidad de insumos médicos necesarios para la atención clínica de los usuarios. Sobre el abastecimiento de suplementos de hierro éstos no pueden ser adquiridos por los CAR considerando que la normativa de salud lo limita, al ser un insumo que el rector en salud debe brindar en al ámbito de sus competencias funcionales.

¿Usted considera que la intervención efectuada por el área de Salud del CAR se ajusta a las necesidades de las niñas, niños y adolescentes y que ésta reconoce sus respectivas particularidades? Explique. Los entrevistados manifestaron que la intervención efectuada por el área de Salud del CAR no se ajusta a las demandas de los usuarios y que ésta no reconoce sus respectivas particularidades, lo que según afirman se debe a las limitaciones presupuestales y a la cantidad de profesionales disponible que se limitan a cumplir con las indicaciones que emite el médico del Primer Nivel de Atención del MINSA.

Finalmente, los entrevistados manifestaron que los manejos de la anemia en los servicios sanitarios se desarrollan con recursos humanos limitados que requieran fortalecer sus capacidades para atender las demandas de los usuarios, por lo que reciben atenciones de salud del Rector, a veces no especializadas y no priorizadas. Los recursos financieros no se ajustan a las necesidades existentes y están limitados a las competencias funcionales de los sectores y al trabajo multisectorial que debe existir en el Estado.

Objetivo específico 5: Analizar el manejo de la anemia en el proceso de reinserción familiar de las niñas, niños y adolescentes acogidos en los Centros de Acogida Residencial del INABIF. Para responder a este objetivo se realizaron las siguientes preguntas:

Usted es conocedor(a) que las niñas, niños y/o adolescentes acogidas en mayoría han sido expuestos a diversos tipo de riesgos (Violencia sexual en el ámbito familiar, Explotación sexual de niñas, niños y adolescentes, Violencia física en el ámbito familiar, Violencia psicológica en el ámbito familiar, Trabajo de niñas, niños y adolescentes en situación de calle o aquel que suponga una afectación de derechos, Vida en calle, Mendicidad, Negligencia o descuido, Conducta de riesgo en la familia de origen, Trata de niñas, niños o adolescentes, Abandono), a partir de ello, tiene conocimiento cómo el CAR evalúa si es adecuado el cuidado y las acciones que se realizan 
para desaparecer las circunstancias que dieron origen a la desprotección y proceda la reintegración familiar-social de la niña, niño y adolescente? Los entrevistados manifestaron que como parte de sus actividades al ingreso de los usuarios al CAR éste inicia las acciones correspondientes para restituir sus derechos, siendo uno de ellos el de "educación", precedido del estado de salud, por lo cual los usuarios son matriculados en la Educación Básica a fin de que prosigan sus estudios en la medida de sus capacidades. Adicionalmente, los usuarios son capacitados en talleres de diverso tipo los que no necesariamente responden a intervenciones individuales dirigidas a que el usuario al ser externado pueda iniciar un emprendimiento, refieren además algunos CAR trabajan con planes generales de intervención educativa. Asimismo, manifiestan que los equipos sociales en algunos casos paralelamente evalúan si en el núcleo familiar o familia extensa han desaparecido las circunstancias que dieron origen a la desprotección y proceda la reintegración familiar-social del menor, para lo cual trabajan en fortalecer los vínculos afectivos de esa familia o la familia extensa.

¿Usted tiene conocimiento de cómo se da el proceso de reintegración familiar en el CAR? Detalle. Los entrevistados manifiestan que tienen conocimiento de cómo se da el proceso de reintegración familiar en el CAR, señalando además que éste se da en cumplimiento de la Ley, en coordinación con las Unidades de Protección Especial mediante el trabajo del equipo social que aborda las particularidades de cada niño a fin de trabajar con las familias cuando van superando las fases señaladas en la normativa y preparando a los usuarios para el egreso.

Adicionalmente, señalan conocer un protocolo implementado por el área social que les permite monitorear el cumplimiento de las familias de origen respecto a las obligaciones con los residentes e iniciar el encargo de trabajar con las familias fortaleciendo sus capacidades y los vínculos afectivos a fin de que la familia se encamine a una crianza saludable y posteriormente el menor se reintegre de manera adecuada.

¿Ha notado si la presencia de algún tipo de anemia en la niña, niño y adolescente podría estar limitando la reintegración familiar - social? Detalle. La mayoría de entrevistadas consideran que la presencia de algún tipo de anemia en los usuarios no estaría limitando la reintegración familiar-social, ya que afirman si alguno de ellos tuviera anemia es recuperado durante su instancia en el CAR. Sin embargo; también refieren que si el niño o adolescente sufrió anemia de manera crónica y ésta afectó el rendimiento escolar si podría influir en la reinserción familiar de manera sostenible ya que su rendimiento intelectual no será el mismo, convirtiéndose en un riesgo. Asimismo, refieren que llevan a cabo "Seguimiento" a los casos, de manera individualizada y minuciosa, pudiendo extenderse ese monitoreo hasta un año dependiendo la casuística.

¿Usted tiene conocimiento si en el CAR las niñas, niños y adolescentes son capacitados o asisten a algún taller de capacitación que pudiera generar algún ingreso económico posterior al egreso? Detalle. Los entrevistados manifestaron que los usuarios son capacitados en talleres dirigidos a prepararlos para una vida laboral una vez que egresen. Algunos de ellos egresan por mayoría de edad fortalecidos en sus capacidades y destrezas otros porque son reintegrados en su núcleo familiar o familia extensa, también fortalecidos en sus capacidades con el objetivo de que puedan aportar económicamente en sus familias. Asimismo, les hacen seguimiento para saber cómo le está yendo e ir encaminando, incentivando y asistiéndolos técnicamente en cómo 
administrar su dinero. Las capacitaciones o talleres en algunos CAR se ajustan a los gustos y preferencias de sus usuarias y en otros las capacitaciones son genéricas y están a cargo de la psicóloga o trabajadora social del CAR.

¿Usted tiene conocimiento si las niñas, niños y adolescentes en el CAR tienen un plan de vida que los ayude a lograr una reintegración familiar-social exitosa? Los entrevistados manifestaron que los usuarios en el CAR tienen un plan de vida que los ayude a lograr una reintegración familiar-social; sin embargo, no pueden asegurar que ésta sea exitosa. Los planes de vida elaborados por los equipos técnicos muchas veces son muy generales o una copia del plan de vida de otro niño no, lo que podría incidir en su reinserción, ya que a pesar de lograr el egreso, podría reingresar debido a múltiples factores (institucionalización, la familia no puede brindarle lo que necesita, etc.).

Finalmente, manifestaron que el proceso de reinserción familiar da inicio con el aspecto de salud, donde se recupera el usuario de la anemia, seguido de lo educativo, posterior a ello se activan las etapas que conllevaran al proceso de reinserción familiar, siendo la última de ellas la preparación para el egreso, dotados de una habilidad técnica que les permita ser independientes económicamente y apoyar a sus familias. Estas actividades programadas en algunos CAR responden a los planes de vida individuales elaborados con la finalidad de prepararlos para el egreso.

De acuerdo al Objetivo general: Analizar el manejo de la anemia, estado de salud y reinserción familiar-social en las niñas, niños y adolescentes acogidos en los Centros de Acogida Residencial del INABIF, los entrevistados manifestaron que el manejo de la anemia preventiva y terapéutica en el lugar de estudio se realiza según MINSA, quienes suministran multimicronutrientes y sulfato ferroso respectivamente. No todos los niveles jerárquicos y distintos profesionales del INABIF conocen a detalle la normativa. Asimismo, los profesionales del ente rector aplican el tratamiento según las especialidades y profesionales. El CAR no adquiere suplementación ante un eventual desabastecimiento. También, manifestaron que el estado de salud de los usuarios respecto de los determinantes: entorno físico, estilo de vida, biología humana y servicios sanitarios resultan beneficiosos para ellos, cuando éstos tienen anemia, aunque identifican debilidades en la alimentación debido a la carencia de un profesional especializado que haga el acompañamiento técnico y en la actividad física al dirigir las mismas sólo a los mayores de 03 años. Respecto de la biología humana, el equipo de salud no considera las individualidades, ni atiende sus particularidades en el manejo de la anemia. Los Servicios Sanitarios se desarrollan con recursos humanos limitados que requieran fortalecer sus capacidades para atender las demandas de los usuarios, por lo que reciben atenciones de salud del Rector, a veces no especializadas y no priorizadas. Los recursos financieros no se ajustan a las necesidades existentes y están limitados a las competencias funcionales de los sectores y al trabajo multisectorial que debe existir en el Estado. Por último, manifestaron que el proceso de reinserción familiar da inicio con el aspecto de salud, donde se recupera el usuario de la anemia, seguido de lo educativo, posterior a ello se activan las etapas que conllevaran al proceso de reinserción familiar, siendo la última de ellas la preparación para el egreso, dotados de una habilidad técnica que les permita ser independientes económicamente y apoyar a sus familias. 
Luego de la aplicación de la entrevista a profundidad se obtuvieron las siguientes categorías: Manejo de la anemia, Estado de salud, Reinserción familiar y social y Competencia, las tres primeras categorías ya consideradas antes del diagnóstico, las cuales fueron consideradas apriorísticamente, y con la aplicación del instrumento se identificó; la última Competencia resultando ser la variable emergente.

La categoría Competencia, según Chomsky (citado en Tobón, 2006) constituye algo interno, en la línea conductual, donde la competencia transitó hasta llegar a ser considerada un comportamiento efectivo, y hoy en día hay un sólido modelo conductual de las competencias, que, aunque ha trascendido el esquema de estímulo-respuesta, sigue basándose en el comportamiento observable, efectivo y verificable, confluyendo entonces así el desempeño dentro de la competencia.

McClelland y Mertens (citados en Ministerio de Salud, 2011) refirieron que las investigaciones que realizaron estuvieron orientados a identificar criterios científicos explicativos de la eficiencia del recurso humano en el desempeño laboral, reconociendo en ellos una alta valoración como poseedores del conocimiento y capacidad de replicarlo en pos de alcanzar los objetivos de la organización. Estas investigaciones, sentaron las bases para la implementación de las competencias en la gestión del potencial humano, convirtiéndose en el último decenio en una ventaja estratégica para ordenar y dirigir las aptitudes de las personas con los fines institucionales.

Lograr este alineamiento, exige además de la formación técnica/especifica propia de una determinada profesión u ocupación, las capacidades genéricas asociadas al comportamiento y a la conducta, también llamadas habilidades blandas. Chiavenato (2009) señaló que: La competencia es una recopilación de comportamientos y habilidades que algunas personas u organizaciones dominan mejor que otras, y que las hace eficaces y competitivas ante una situación determinada. La competencia constituye un acumulado de conocimientos, experiencia, prácticas, comportamientos y tipos de razonamientos adquiridos en función de la experiencia; es decir, es el conjunto de conocimientos, habilidades, actitudes e intereses que distinguen a personas y organizaciones. Así, las competencias son observables en situaciones cotidianas de trabajo y en situaciones de prueba, incorporando de esta manera el desempeño.

Los entrevistados manifestaron que el manejo de la anemia en los CAR se realiza según lo indicado por el personal de salud de los Establecimientos de Salud del MINSA, con recursos humanos que no estarían capacitados en cómo manejar la anemia y el estado de salud de los mismos; no tienen la experiencia requerida, a lo que se suma falta de interés por satisfacer las demandas de los usuarios, lo que en suma resulta en la necesidad de fortalecimiento de capacidades, formación especializada y experiencia a adquirir en los recursos humanos a cargo de la población.

\section{Conclusiones y Recomendaciones}

El manejo de la anemia en los CAR tanto preventiva como terapéutica se realiza tomando en cuenta la normativa del MINSA, quienes son responsables del suministro. La citada norma no 
es aplicada en los mismos términos por todos los profesionales de la salud. Se recomienda al MINSA estandarizar la aplicación de la normativa para la prevención y atención de la anemia a fin de que todos los profesionales de la salud cumplan con suministrar los suplementos preventivos y terapéuticos el tiempo que fisiológicamente indica la misma.

El entorno físico, como el medio ambiente social del CAR es beneficioso para el estado de salud de los usuarios cuando éstos tienen anemia. Se recomienda al INABIF garantizar asegurar que todos los CAR cuenten con las condiciones de entorno: físico y medio ambiente social que satisfagan las necesidades de los usuarios.

El manejo de la anemia respecto de la biología humana y el sistema inmunológico de los usuarios, no es abordado en los CAR según sus individualidades y atendiendo sus particularidades. Se recomienda al INABIF el manejo de la anemia tenga en cuenta las individualidades de los usuarios respecto de la biología humana y el sistema inmunológico de los mismos, para lo cual los equipos técnicos de los CAR deben estar capacitados y especializados para responder y atender sus necesidades particulares, considerando que un manejo inadecuado sobre todo a edades tempranas puede no evitar pérdida del capital humano.

El manejo de la anemia en los servicios sanitarios se desarrolla con recursos humanos limitados que requieran fortalecer sus capacidades para atender las demandas especializadas de los usuarios. Los recursos financieros no se ajustan a las necesidades existentes y están limitados a las competencias funcionales de los sectores y al trabajo multisectorial que debe existir en el Estado. Se recomienda al INABIF los CAR cuenten con equipos técnicos completos y fortalecidos en sus competencias a fin de atender las demandas especializadas de los usuarios, ya que la ausencia de alguno de ellos hace que recurran a las atenciones en MINSA que no son especializadas en el marco del Sistema Integral de Salud.

El manejo de la anemia en el proceso de reinserción familiar da inicio con la recuperación del usuario de la anemia, seguido de lo educativo, donde a partir de los planes de vida individuales son preparados para el egreso y posterior reinserción familiar, dotados de una habilidad técnica que les permita ser independientes económicamente y apoyar a sus familias. Se recomienda al INABIF que los usuarios de los CAR reciban capacitaciones y adiestramientos acordes a sus planes de vida y sostenibles, a fin de que de manera exitosa puedan ser independientes económicamente si son mayores de edad y apoyar a sus familias. Si esto no sucede la probabilidad del reingreso se convierte en un riesgo.

\section{Referencias Bibliográficas}

Acevedo, M. y Alba F. (1986). El proceso de la entrevista conceptos y modelos (3 ed.). México DF: LIMUSA.

Arredondo, A. (1992). Analysis and Reflection on Theoretical Models of the Health - Disease Process. Cad Saude Públ., Río de Janeiro, 8 (3), 254-261. 
Baena, G. (1985) Manual para elaborar trabajos de investigación documental, México: Edit. Mexicanos Unidos, 1985. 4a ed., 2a reimpreso.

Balcázar, P. Gonzales-Arratia, N. Gurrola, G. y Moysén, A. (2013) Investigación cualitativa, Libros electrónicos y capítulos de libros. Recuperado: http://repositorio.minedu.gob.pe/handle/123456789/4641

Cadet, M. (2018). Iron Deficiency Anemia: AClinical Case Study. March-April 2018. Vol. 27/No. 2.

Carrasco, D., (2019). Metodología de la Investigación Científica (2a. ed.). Lima: Editorial San Marcos.

Chaverra, B. Gaviria, F. y González, V. (2019). "El estudio de caso como alternativa metodológica e la investigación en educación física, deporte y actividad física, conceptualización y aplicación". Retos: nuevas perspectivas de educación física, deporte y recreación, (35), 422-427. Recuperado de http://cutt.ly/Ta3hsyB

Comité Nacional de Hematología, Oncología y Medicina Transfusional, Comité Nacional de Nutrición. Iron Deficiency and Iron Deficiency Anemia. Guideline for Prevention, Dagnosis and Treatment. Executive Summary. Arch. Argent Pediatr 2017;115 Supl 4:s68s82.

Convención de las Naciones Unidas (1989). Convención sobre los Derechos del Niño.

CEPLAN (2011) Plan Bicentenario. El Perú hacia el 2021.

Decreto Legislativo $\mathrm{N}^{\circ}$ 1297/2016, 29 de diciembre, Decreto Legislativo para la protección de niñas, niños y adolescentes sin cuidados parentales o en riesgos de perderlos. Diario el peruano, pp. 610476. Lima, Perú.

Decreto Supremo No 001/2018-MIMP, 10 de febrero. Aprueban Reglamento del D. Leg. No 1297 , Decreto Legislativo para la Protección de las niñas, niños y adolescentes sin cuidados parentales o en riesgo de perderlos. Diario el peruano, pp. 21

Denzin, N., \& Lincoln, Y. (2005). The Sage Handbook of Qualitative Research. London, Inglaterra: Sage.

Díaz, A. et al (2015) Health and nutrition of indigenous and nonindigenous children in the Peruvian Amazon. Rev. Panamericana de Salud Pública. 2015:38(1)49-56.

Fahima, H. et al (2016). Evaluation of iron deficiency anemia in infancy. Menoufia Medical Journal, vol.29, no 2, 2016, p. 269. Gale Academic Onefile.

Fernández-Daza, M. (2018). Family reintegration in Latin America. Saúde Soc. Sao Paulo, v. 27. n.i, p.268-289, 2018.

Gonzáles, J. (2012). Observatorio Juvenil Interactivo de Transformación Social, San José, Costa Rica.

Hernández, R., Fernández, C., y Baptista, P. (2000). Metodología de la investigación. Ciudad de México: Mc Graw Hill.

Hernández, R., Fernández, C., y Baptista, M. (2014). Metodología de la investigación. Ciudad de México: Mc Graw Hill.

Instituto Nacional de Estadística e Informática. (2018). Lima.

Kleinman, A. (1980). Patients and healers in the context of culture. Berkeley, CA: University of California Press.

López M. et al. (2014). Characteristics and Development of Foster Care in Two Countries with a Strong Tradition of Residential Care: Spain and Portugal. Bogotá, Colombia.

Martínez, M. (2006) La investigación cualitativa (Síntesis Conceptual) Revista IIPSI. Facultad de Psicología UNMSM. Recuperado de: https://bit.ly/38tjAh3

Esta obra se comparte bajo la licencia Creative Common Atribución-No Comercial 4.0 International (CC BY-NC 4.0) Revista de la Universidad Internacional del Ecuador. URL: https://www.uide.edu.ec/ 
Martínez, F. (1987) El método inductivo. Universidad Autónoma de Nuevo León. Recuperado: http://eprints.uanl.mx/5599/1/1080071376.PDF

Ministerio de Desarrollo e Inclusión Social (2016). Lineamientos "Primero la Infancia"

Ministerio de la Mujer y Poblaciones Vulnerables 2018). Reglamento del Decreto Legislativo $N^{\circ}$ 1297 para la protección de las niñas, niños y adolescentes sin cuidados parentales o en riesgo de perderlos. Lima, Perú.

Ministerio de la Mujer y Poblaciones Vulnerables (2012). Plan Nacionalde Acción por la Infancia y la Adolescencia 2012-2021. Lima.

Ministerio de Salud (2017). Plan Nacional para la reducción y control de la anemia Materno Infantil y la Desnutrición Crónica Infantil en el Perú: 2017-2021. Lima.

Ministerio de Salud (2017). Norma Técnica - Manejo Terapéutico y Preventivo de la anemia en niños, adolescentes, mujeres gestantes y puérperas. Perú 2017.

Ministerio de Salud - Instituto Nacional de Salud (2018). Programa de entrenamiento en salud pública dirigido a personal de servicio militar voluntario. Guía del participante. Lima.

Naupas, H. et al. (2014) Metodología de la investigación Cuantitativa y Cualitativa y redacción de tesis. Ediciones de la Univ. Transversal. 4ta ed. Bogotá - Colombia.

Organización Panamericana de la Salud (2019). Tendencias de la Salud en las Américas. Indicadores Básicos 2019.

Organización de las Naciones Unidas (1959). Declaración de los Derechos del Niño.

Organización de las Naciones Unidas (2010) Directrices sobre las modalidades alternativas de cuidado de los niños. RES/64/142.

Pavo et. al (2016) Anemia en la edad pediátrica. Madrid, España.

Secretaría de Salud, 2010. Prevención, Diagnóstico y Tratamiento de la Anemia por Deficiencia de Hierro en Niños y Adultos. México.

Presidencia del Consejo de Ministros (2018). Plan Multisectorial de Lucha contra la Anemia. Lima.

Pérez, M. (2016). Multimicronutrientes versus sulfato ferroso: Un meta análisis de los efectos de los tratamientos para la reducción de anemia infantil, en el marco de la Meta 1 del Objetivo 2 de los ODS al 2030. Lima, Perú.

Quecedo, R. y Castaño, C. (2002) Introducción a la metodología de investigación cualitativa. Recuperado de: https://bit.ly/3e0DyRx.

Red Latinoamericana de Acogimiento Familiar (2015). Decálogo de Estándares de Derechos Humanos aplicables a Niños, Niñas y Adolescentes que se encuentran en proceso de evaluación e instituciones que se ocupan de la protección especial de derechos.

Ricoy, C. (2006) Contribución sobre los paradigmas de investigación Educação. Revista do Centro de Educação, vol. 31, núm. 1, 2006, Universidade Federal de Santa Maria, RS, Brasil.

Rodríguez, G., Gil, J. y García, E. (1999) Metodología de la investigación cualitativa, Universidad de Cádiz -España

Ruiz, J. (1999). Metodología de la investigación cualitativa. Bilbao: Universidad de Deusto. Recuperado de: https://bit.ly/2W35SfZ.

Sánchez, H. y Reyes, C. (2006). Metodología y Diseños en la Investigación Científica. Lima: Editorial Visión Universitaria.

Solís, I. (2003). El análisis documental como eslabón para la recuperación de información y los servicios. Recuperado: http://bit.ly/2Z0f4Dw

Esta obra se comparte bajo la licencia Creative Common Atribución-No Comercial 4.0 International (CC BY-NC 4.0) Revista de la Universidad Internacional del Ecuador. URL: https://www.uide.edu.ec/ 
Sulayyim, H. et al. BMC Pediatrics (2019) An assessment for diagnostic and therapeutic modalities for management of pediatric Iro defficiency Anemia in Saudi Arabia: a crossectional study. 19:314

Ugalde N. y Balbastre F. (2013). Investigación Cuantitativa e Investigación Cualitativa: Buscando las ventajas de las diferentes metodologías de investigación. Recuperado de http://revistas.ucr.ac.cr/index.php/economicas/article/view/12730/11978

Viquez, M. (2014). Abordaje de la Anemia por Deficiencia de Hierro en niños y niñas de 6 a 24 meses de edad de Costa Rica. Caja Costarricense de Seguro Social.

WHO (2011). Concentraciones de hemoglobina para diagnosticar la anemia y evaluar su gravedad. Ginebra, Organización Mundial de la Salud, 2011 (WHO/NMH/NHD/MNM/11.1).

WHO (2015). The global prevalence of anemia in 2011. Geneva: World Health Organization.

WHO (2006) Constitución de la Organización Mundial de la Salud. Documentos básicos, suplemento de la $45^{\circ}$ edición, octubre de 2006. 